\title{
Bottleneck identification and alleviation in a blocked serial production line with discrete event simulation: A case study
}

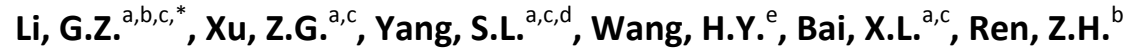 \\ ${ }^{a}$ State Key Laboratory of Robotics, Shenyang Institute of Automation, Chinese Academy of Science, Shenyang, P.R. China \\ ${ }^{b}$ School of Mechanical Engineering and Automation, Northeastern University, Shenyang, P.R. China \\ 'Institutes for Robotics and Intelligent Manufacturing, Chinese Academy of Sciences, Shenyang, P.R. China \\ dUniversity of Chinese Academy of Sciences, Beijing, P.R. China \\ e Shanghai Aerospace Chemical Engineering Institute, Huzhou, P.R. China
}

\section{A B S T R A C T}

Aiming at the gap between theoretical research and practical application in the production bottleneck field, we apply five bottleneck identification methods in a serial production line in aerospace industry based on discrete event simulation and Plant Simulation software, meanwhile discuss the influence of the bottleneck machine quantity on the system performance. This paper evaluated the practicability, accuracy and limitation of various bottleneck identification methods at the practical level. The results of the bottleneck alleviation manifest that increasing the number of bottleneck machines can effectively improve the system performance, but the more machine quantity, the smaller performance improvement. More importantly, the paper studies the influence mechanism and function relationship of the bottleneck machine quantity on the maximum completion time from an interesting actual phenomenon for the first time. The function obtains the condition that the maximum completion time achieves the minimum. The research and conclusion of this paper have essential reference significance for production guidance and theoretical research, and can also contribute to narrow the gap between theory and application of the production bottleneck field.
\end{abstract}

\section{ARTICLE INFO}

Keywords:

Serial production line;

Bottleneck identification;

Bottleneck alleviation;

Discrete event simulation;

Plant Simulation;

Case study

*Corresponding author:

2486541829@qq.com

(Li, G.Z.)

Article history:

Received 15 January 2020

Revised 10 July 2020

Accepted 13 July 2020

(C) 2020 CPE, University of Maribor. All rights reserved.

\section{References}

[1] Watson, K.J., Blackstone, J.H., Gardiner, S.C. (2007). The evolution of a management philosophy: The theory of constraints, Journal of Operations Management, Vol. 25, No. 2, 387-402, doi: 10.1016/i.jom.2006.04.004.

[2] Wang, Y., Zhao, Q., Zheng, D. (2005). Bottlenecks in production networks: An overview, Journal of Systems Science and Systems Engineering, Vol. 14, No. 3, 347-363, doi: 10.1007/s11518-006-0198-3.

[3] Sims, T., Wan, H.-D. (2017). Constraint identification techniques for lean manufacturing systems, Robotics and Computer-Integrated Manufacturing, Vol. 43, 50-58, doi: 10.1016/j.rcim.2015.12.005.

[4] Yan, H.-S., An, Y.-W., Shi, W.-W. (2010). A new bottleneck detecting approach to productivity improvement of knowledgeable manufacturing system, Journal of Intelligent Manufacturing, Vol. 21, No. 6, 665-680, doi: 10.1007 /s10845-009-0244-3.

[5] Scholz-Reiter, B., Windt, K., Liu, H. (2010). Modelling dynamic bottlenecks in production networks, International Journal of Computer Integrated Manufacturing, Vol. 24, No. 5, 391-404, doi: 10.1080/0951192X.2010.511655.

[6] Li, L., Chang, Q., Ni, J., Biller, S. (2009). Real time production improvement through bottleneck control, International Journal of Production Research, Vol. 47, No. 21, 6145-6158, doi: 10.1080/00207540802244240.

[7] Shi, W.-W., Yan, H.-S. (2006). Method of shifting bottleneck analysis in knowledge-oriented manufacturing system, Computer Integrated Manufacturing Systems, Vol. 12, No. 2, 271-279, doi: 10.13196/j.cims.2006.02.113. shiww.020. 
[8] Li, X., Yuan, Y., Sun, W., Feng, H. (2016). Bottleneck identification in job-shop based on network structure characteristic, Computer Integrated Manufacturing Systems, Vol. 22, No. 4, 1088-1096, doi: 10.13196/i.cims.2016. $\underline{04.023}$.

[9] Liu, M., Tang, J., Ge, M., Jiang, Z., Hu, J., Ling, L. (2009). Dynamic prediction method of production logistics bottleneck based on bottleneck index, Chinese Journal of Mechanical Engineering, Vol. 22, No. 5, 710-716, doi: 10.3901/CJME.2009.05.710.

[10] Zhou, F.L., Wang, X., He, Y.D., Goh, M. (2017). Production lot-sizing decision making considering bottle-neck drift in multi-stage manufacturing system, Advances in Production Engineering \& Management, Vol. 12, No. 3, 213-220, doi: 10.14743/apem2017.3.252.

[11] Zhou, J., Deng, J.X., Huang, P.L., Liu, Z.Q., Ai, X. (2006). Integrated analysis method: Visual modelling, simulation, diagnosis and reduction for bottleneck processes of production lines, Iranian Journal of Science \& Technology, Transaction B, Engineering, Vol. 30, No. B3, 363-375.

[12] Pehrsson, L., Ng, A.H.C., Bernedixen, J. (2016). Automatic identification of constraints and improvement actions in production systems using multi-objective optimization and post-optimality analysis, Journal of Manufacturing Systems, Vol. 39, 24-37, doi: 10.1016/i.jmsy.2016.02.001.

[13] Li, H.Y., Gui, C., Xiao, K. (2018). Simulation of multivariate scheduling optimization for open production line based on improved genetic algorithm, International Journal of Simulation Modelling, Vol. 17, No. 2, 347-358, doi: 10.2507/IJSIMM17(2)C09.

[14] Betterton, C.E., Silver, S.J. (2012). Detecting bottlenecks in serial production lines-a focus on interdeparture time variance, International Journal of Production Research, Vol. 50, No. 15, 4158-4174, doi: 10.1080/00207543.2011. $\underline{596847 .}$.

[15] Hajmirfattahtabrizi, M., Song, H. (2019). Investigation of bottlenecks in supply chain system for minimizing total cost by integrating manufacturing modelling based on MINLP approach, Applied Sciences, Vol. 9, No. 6, Article No. 1185, doi: 10.3390/app9061185.

[16] Kang, Y., Ju, F. (2019). Integrated analysis of productivity and machine condition degradation: Performance evaluation and bottleneck identification, IISE Transactions, Vol. 51, No. 5, 501-516, doi: 10.1080/24725854.2018. 1494867.

[17] Kefeli, A., Uzsoy, R. (2016). Identifying potential bottlenecks in production systems using dual prices from a mathematical programming model, International Journal of Production Research, Vol. 54, No. 7, 2000-2018, doi: $10.1080 / 00207543.2015 .1076182$.

[18] Lei, Q., Li, T. (2017). Identification approach for bottleneck clusters in a job shop based on theory of constraints and sensitivity analysis, Proceedings of the Institution of Mechanical Engineers, Part B: Journal of Engineering Manufacture, Vol. 231, No. 6, 1091-1101, doi: 10.1177/0954405415583884.

[19] Lawrence, S.R., Buss, A.H. (1994). Shifting production bottlenecks: Causes, cures, and conundrums, Production and Operations Management, Vol. 3, No. 1, 21-37, doi: 10.1111/j.1937-5956.1994.tb00107.x.

[20] Kikolski, M. (2016). Identification of production bottlenecks with the use of Plant Simulation software, Engineering Management in Production and Services, Vol. 8, No.4, 103-112, doi: 10.1515/emj-2016-0038.

[21] Roser, C., Nakano, M., Tanaka, M. (2001). A practical bottleneck detection method, In: Proceedings of the 2001 Winter Simulation Conference, Arlington, USA, 949-953, doi: 10.1109/WSC.2001.977398.

[22] Sengupta, S., Das, K., VanTil, R.P. (2008). A new method for bottleneck detection, In: Proceedings of the 2008 Winter Simulation Conference, Miami, USA, 1741-1745, doi: 10.1109/WSC.2008.4736261.

[23] Zhang, R., Wu, C. (2012). Bottleneck machine identification method based on constraint transformation for job shop scheduling with genetic algorithm, Information Sciences, Vol. 188, 236-252, doi: 10.1016/j.ins.2011.11.013.

[24] Li, G., Xu, Z., Ren, Z. (2019). Simulation and optimization of plant production takt, In: Proceedings of the 2019 International Conference on Modeling, Simulation and Big Data Analysis, Wuhan, China, 98-103, doi: 10.2991/ msbda-19.2019.16.

[25] Ojstersek, R., Lalic, D., Buchmeister, B. (2019). A new method for mathematical and simulation modelling interactivity: A case study in flexible job shop scheduling, Advances in Production Engineering \& Management, Vol. 14, No. 4, 435-448, doi: 10.14743/apem2019.4.339.

[26] Zhang, Z., Wang, X., Wang, X., Cui, F., Cheng, H. (2019). A simulation-based approach for plant layout design and production planning, Journal of Ambient Intelligence and Humanized Computing, Vol. 10, No. 3, 1217-1230, doi: 10.1007/s12652-018-0687-5.

[27] Jia, Y., Tian, H., Chen, C., Wang, L. (2017). Predicting the availability of production lines by combining simulation and surrogate model, Advances in Production Engineering \& Management, Vol. 12, No. 3, 285-295, doi: 10.14743/ apem2017.3.259.

[28] Khalid, R., Nawawi, M.K.M., Kawsar, L.A., Ghani, N.A., Kamil, A.A, Mustafa, A. (2013). A discrete event simulation model for evaluating the performances of an M/G/C/C state dependent queuing system, Plos One, Vol. 8, No. 4, Article No. e58402, doi: 10.1371/journal.pone.0058402.

[29] Ištoković, D., Perinić, M., Doboviček, S., Bazina, T. (2019). Simulation framework for determining the order and size of the product batches in the flow shop: A case study, Advances in Production Engineering \& Management, Vol. 14, No. 2, 166-176, doi: 10.14743/apem2019.2.319.

[30] Silva, M. (2018). On the history of discrete event systems, Annual Reviews in Control, Vol. 45, 213-222, doi: 10.1016/j.arcontrol.2018.03.004.

[31] de Sousa Junior, W.T., Montevechi, J.A.B., de Carvalho Miranda, R., Campos, A.T. (2019). Discrete simulationbased optimization methods for industrial engineering problems: A systematic literature review, Computers \& Industrial Engineering, Vol. 128, 526-540, doi: 10.1016/j.cie.2018.12.073. 


\title{
Identifikacija in odprava ozkih grl v blokirani serijski proizvodni liniji s simulacijo diskretnih dogodkov: Študija primera
}

\author{
Li, G.Z. ${ }^{a, b, c,{ }^{*}}$, Xu, Z.G. ${ }^{a, c}$, Yang, S.L. ${ }^{a, c, d}$, Wang, H.Y. ${ }^{\text {, }}$, Bai, X.L. ${ }^{a, c}$, Ren, Z.H. ${ }^{b}$ \\ ${ }^{\text {a }}$ State Key Laboratory of Robotics, Shenyang Institute of Automation, Chinese Academy of Science, Shenyang, P.R. China \\ ${ }^{\mathrm{b}}$ School of Mechanical Engineering and Automation, Northeastern University, Shenyang, P.R. China \\ 'Institutes for Robotics and Intelligent Manufacturing, Chinese Academy of Sciences, Shenyang, P.R. China \\ dUniversity of Chinese Academy of Sciences, Beijing, P.R. China \\ ${ }^{\text {e}}$ Shanghai Aerospace Chemical Engineering Institute, Huzhou, P.R. China
}

\section{POVZETEK}

Glede na vrzel med teoretičnimi raziskavami in praktično uporabo na področju ozkih grl v proizvodnji, uporabimo pet metod identifikacije ozkih grl na serijski proizvodni liniji za vesoljsko industrijo, ki temeljijo na simulaciji diskretnih dogodkov in programski opremi Plant simulation, hkrati pa razpravljamo o vplivu ozkega grla, zaradi števila strojev, na delovanje sistema. V prispevku je bila ocenjena izvedljivost, natančnost in omejitev različnih načinov prepoznavanja ozkih grl na praktični ravni. Rezultati odpravljanja ozkih grl kažejo, da lahko povečanje števila strojev, zaradi katerih se pojavi ozko grlo, učinkovito izboljša delovanje sistema, vendar pa večje število strojev hkrati pomeni manjše izboljšanje zmogljivosti. Še pomembneje je, da članek na podlagi zanimivega dejanskega pojava prvič preučuje mehanizem vpliva in funkcijsko odvisnost ozkega grla zaradi števila strojev na največji čas zaključka. Funkcijski pogoj je, da najdaljši čas zaključka doseže minimum. Raziskave in sklepi tega prispevka so bistvenega referenčnega pomena za vodenje proizvodnje in za teoretične raziskave ter lahko prispevajo tudi k zmanjšanju vrzeli med teorijo in prakso na področju ozkega grla v proizvodnji.

\section{PODATKI O ČLANKU}

Ključne besede:

Serijska proizvodna linija; Identifikacija ozkega grla; Zmanjšanje ozkih grl;

Simulacija diskretnih dogodkov;

Plant simulation;

Študija primera

*Kontaktna oseba: 2486541829@qq.com (Li, G.Z.)

Zgodovina članka:

Prejet 15. januarja 2020

Popravljen 20. julija 2020

Sprejet 13. julija 2020

(c) 2020 CPE, University of Maribor. All rights reserved. 